\title{
Luxe et virtualité: le vertige du contrôle
}

\author{
Didier Tsala Effa
}

Résumé: Alors que la communication des marques de luxe a été dotée depuis toujours de supports spécifiques (boutiques particulières, pages de magazines spécifiques, codes publicitaires propres) à l'écart d'autres supports médiatiques plus génériques, cette communication se doit désormais de se définir aussi ses nouveaux codes, pour construire sa spécificité dans les nouveaux supports virtuels, à commencer par sa présence sur internet. Avec la contrainte de son exclusivité, à savoir, éviter la banalité, cultiver son exception. Non pas qu'il faille inventer de nouveaux contenus, «le luxe c'est le luxe» dira-t-on, mais à l'évidence, il s'agit de s'approprier autrement cet espace dans sa particularité, de manière à dire toujours le luxe sans confusion. Un paradoxe. La problématique qui s'ensuit à l'évidence est celle de la préservation d'un statut. Face à une telle nécessité de réorganisations, face aux nouvelles contraintes, de quelle manière les marques de luxe opèrent-elles pour maintenir leur essence? Selon quelles modalités? Notre travail, en partant de différents sites de marques luxe (mode et accessoires, joaillerie, horlogerie), est de mettre en avant le vertige du contrôle comme le cœur de la stratégie de ces marques.

Mots-clefs: marque de luxe; virtualité; supports médiatiques.

Abstract: Luxury and virtuality: the vertigo of control. - The communication of luxury brands had once counted on specific media (special shops, specific pages of magazines, its own advertising codes) distant from other more generic media vehicles, but nowadays such communication must define its new codes, in order to build its specificity in the new virtual media, starting with its presence on the internet, with the constraint of its exclusivity, i.e., to avoid banality and cultivate its exception. Not that we should invent new content, "the luxury is the luxury," one could say, but obviously it is about the appropriation of this space in its particularity, so it can convey luxury without confusion. A paradox. The problem that follows this evidence is that of the preservation of a status. Facing such a need for reorganization, given the new constraints, how do the luxury brands operate to maintain their essence? According to what modalities? Our research, based on various luxury brand websites (fashion and accessories, jewelry, watchmaking), is to highlight the vertigo of controlling as the heart of the strategy of these brands.

Keywords: luxury brand; virtuality; media vehicles. 


\section{Une ambiguiité: distinction vs démocratisation}

Aborder la question de la communication du luxe revient presque toujours à traiter d'une ambiguïté, et avec un grand écart, entre d'un côté le souci de préserver la ligne de distance nécessaire qui confère au luxe sa distinction, parmi les autres produits destinés à la consommation et de l'autre un désir parfaitement légitime de démocratisation. En effet, on ne peut plus feindre de méconnaître au luxe cette autre dimension qui fait qu'il est aussi et même de plus en plus prioritairement des parts de marché. Pour le dire de manière directe, alors que demeure toujours la nostalgie du luxe comme reflet exclusif de la Chevalerie, s'est imposée définitivement la nécessité de le démocratiser, non pas tant d'abord pour permettre au plus grand nombre d'y accéder, mais parce qu'il en va de la survie même de l'idée du luxe. Il y est question d'économie: nul ne songerait à contester que le luxe a toujours été, y compris par le passé, sous-tendu par des contraintes économiques. Or la puissance économique aujourd'hui n'est plus, tant que cela, ce qui est réservé à certains, mais devient au moins ce que tout un chacun est capable d'accumuler pour se permettre quelque chose de manière exceptionnelle. II en est ainsi des modes de consommation du luxe, par exemple cette description proposée par Benoît Heilbrun dans une orientation éminemment marketing, qui envisage le luxe comme «ce que l'on se permet de s'offrir de manière exceptionnelle». ${ }^{1}$ Autrement dit, s'il fallait chercher une raison à la communication du luxe, elle serait assurément de cet ordre. Auparavant exclusif, du fait d'une assimilation un peu trop rapide des classes sociales à tel accès particulier à la richesse, le luxe, en parallèle avec l'éclosion de la consommation de masse, s'est démocratisé, avec une accélération particulièrement intense du fait du développement des moyens de communication virtuels. Tel est notre point de départ.

\section{Le luxe sur internet}

Alors qu'elle a été dotée depuis toujours de supports spécifiques et exclusifs (boutiques particulières, pages de magazines spécifiques, codes publicitaires propres) à l'écart d'autres supports médiatiques plus génériques, la communication des marques de luxe se doit désormais de construire aussi sa spécificité pour continuer à tenir son rang dans les supports virtuels, à commencer par sa présence sur internet; avec la contrainte d'éviter la banalité, et de continuer à cultiver l'exception. Non pas qu'il faille inventer de nouveaux contenus, «le luxe c'est le luxe» dira-t-on, mais à l'évidence, il s'agit de s'approprier opportunément internet dans sa particularité, de manière à dire toujours le luxe sans confusion. Un paradoxe.

1 Heilbrun Benoit, "Le luxe est mort, vive le luxe! Le marché du luxe à l'aune de la démocratisation», in Assouly Olivier, Le luxe. Essai sur la fabrique de l'ostentation, Paris, Édition du regard, 2005, p.365 
Pourtant, s'il est un espace qui s'accommode de confusions, tant les possibilités créatives y sont étendues, c'est bien le support internet. Non seulement les produits de luxe sont disséminés à travers divers sites génériques (sites promotionnels, sites de distribution), mais en outre, les marques de luxe, pour assurer leur présence sur ce support, se doivent d'autre part de composer avec la nécessité d'occuper aussi cet espace comme un lieu de vente, ce qui constitue une deuxième rupture, eu égard aux exigences d'exclusivité du luxe; on ne vend pas un produit de luxe comme tout autre produit. D'entrée, la question qui se pose est celle des stratégies empruntées?

Par notre exposé, en partant d'une observation de plus de 150 sites internet des marques de luxe, tous produits confondus, la mode, la joaillerie, les montres, les voitures de luxe ainsi que les vins et spiritueux, notre idée est de faire un premier point sur l'existant.

\section{Le luxe: un ordre discriminatoire}

Notre hypothèse est pour le moins dogmatique. Quelles qu'en sont les alertes et quelles qu'en sont les offensives, notamment celles développées par les marketeurs, qui tendent à militer de plus en plus fortement pour un effacement progressif des limites entre les marques de luxe et les marques de produits de grande consommation, le luxe en plus d'être aspirationnel, dans la mesure où il invite toujours à contribuer à un univers de légende et de valeurs comme beaucoup d'autres marques, se distingue d'abord en cela qu'il est toujours mû par un ordre discriminatoire. En réalité, on n'y accède que parce qu'on en connaît les véritables codes: un vrai enjeu et un vrai débat entretenu par ailleurs par les créateurs eux-mêmes; par exemple Pierre Bergé, Président de la prestigieuse Fondation Yves Saint-Laurent qui tient fermement à cette part exclusive : «Assigner au luxe une histoire qui soit la sienne, c'est en concevoir d'autres expressions sans pour autant abandonner le luxe à l'anarchie des créateurs sans mesure ou à un mercantilisme sans imagination »².

Cette position est la nôtre, afin de questionner la manière dont les marques de luxe procèdent pour continuer à maintenir leur efficience sur un support de communication des plus démocratisés tel que l'est internet.

\section{Les clichés d'une magnification exceptionnelle}

Dès l'abord, lorsqu'on engage une exploration, même la plus minimale, des sites internet des marques de luxe, on n'échappe pas aux clichés les plus évidents de ce qu'il est habituel d'imaginer d'une marque de luxe: une magnification exceptionnelle avec pour incidence la négation de la banalité et du commun. Les informations ne sont pas de simples énoncés pour présenter et décrire les produits ou la marque, ce sont de petites histoires; les mannequins, lorsqu'ils sont présents, opèrent comme des égéries plutôt que

2 Pierre Bergé, «Préface», in Assouly, op. cit. p.11. 
comme de simples figures publicitaires; quant aux produits, il s'agit surtout des objets et même des objets d'art. La technique y participe aussi par ses prouesses, avec des sites en flash et des défilés dynamiques. En somme sur ces sites internet, tout ce qui s'offre à l'internaute n'a d'abord de justification que parce qu'il donne l'impression de se justifier d'abord d'un supplément (d'âme?). Il en est également des modalités qui sous-tendent l'expression de ces marques en tant que données à voir. Tout opère sous le prisme d'un miroir grossissant, avec des effets d'échelle ; I'argumentaire est laudateur, avec des termes hyperboliques, qui relèvent du merveilleux, du fantastique, avec des objets agrandis, des illustrations «XXL». Au final, à l'endroit où tout autre site en aurait profité pour militer à faveur d'un accès le plus réaliste à ses produits, permettant au plus grand nombre, grâce au support internet, de découvrir, enfin! ce qu'est véritablement le luxe, on observe pour cette communication, un désir d'inscrire toujours de fait une couche médiatique supplémentaire.

\title{
Une entorse sémiosique
}

L'enjeu de signification se résout alors à ce qu'il serait évident de dénommer comme une entorse sémiosique, avec en vue la recherche d'une véritable prise de contrôle sur toute manifestation signifiante en question. En d'autres termes la question qui est posée est celle de l'inscription d'une particularité dans le dispositif sémiotique. Et nous ne sommes pas loin de ce que Hjelmslev appelle la sémiotique connotative. À l'inverse de la sémiotique habituelle où le plan du continu et le plan du contenu ne se définissent que par opposition et relativement l'un par rapport à l'autre, le sémiotique connotative, selon Hjelmslev est une sémiotique où, avant même d'entrer dans le processus sémiosique, le plan de l'expression et le plan du contenu sont déjà des sémiotiques:

\begin{abstract}
«Nous entendons par [sémiotiques dénotatives] dont aucun des plans n'est une sémiotique. Il nous reste à démontrer, en élargissant encore notre perspective, qu'il existe aussi des sémiotiques dont le plan de l'expression est une sémiotique et aussi d'autres dont le plan du contenu est une sémiotique. Nous appellerons les premières sémiotiques connotatives et les secondes métasémiotiques. Comme le plan de l'expression et le plan du contenu ne se définissent que par opposition et relativement l'un par rapport à l'autre, il s'ensuit que les définitions proposées ici de sémiotique connotative et métasémiotique ne sont que les définitions 'réalistes' provisoires auxquelles on ne peut même pas accorder de valeur opérationnelle» ${ }^{3}$
\end{abstract}

Bien évidemment, il y aurait lieu de discuter plus longuement les considérations supplémentaires ici énoncées par Hjelmslev. Ce qui ressort est la prédominance, voire l'intrusion dans chaque formant sollicité pour porter le discours du luxe, d'un élément qui en suppose toujours déjà préalablement le parti pris idiomatique. Par exemple, ceci

3 Hjelmslev Louis, Prolégomènes à une théorie du langage, Paris, Minuit, 1971, p.144. 
est visible dès l'accès même du site internet ; lorsque le site ne s'ouvre pas sur une page dont on ne sait quel chemin emprunter pour la navigation, il impose en général un ordre d'accessibilité, avec un mode et un rythme non maîtrisable a priori: des figures apparaissent et s'imposent toutes seules à la visibilité, et pour certains sites, l'internaute est soumis à une écoute musicale sans aucune possibilité d'en régler le flux. Il se produit ainsi comme un phénomène de désubstantialisation. Ce qui est donné à voir ne l'est pas pour soi, mais parce qu'il est dépossédé de sa substance supposée.

Mais les traductions les plus révélatrices de cette entorse sémiosique sont celles qui s'observent quand on entreprend d'explorer le fond même du discours émis par ces sites pour parler du luxe. D'entrée, l'on est dans l'obligation de recourir à un dispositif cognitif dont on voit qu'il invite à solliciter plus qu'une pure compétence interprétative destinée à faire accéder à un sens construit et disposé là pour être interprété ou co-produit par un énonciataire. En poursuivant le parallèle avec Hjelmslev, ce dispositif cognitif opèrerait en effet comme un connotateur, autrement dit un indicateur qui, face à particularité de tel texte ou de tel discours, en assurerait la traductibilité. C'est le cas ainsi, comme nous venons de le voir, de la prise de contrôle de la navigation sur un site internet par son émetteur, à l'endroit où on s'attendrait pourtant à un accès des plus instantanés.

\section{Quatre formes de contenus: la légende, le documentaire, I'éloge, la chronique}

Les discours tenus par les sites internet des marques de luxe se caractérisent par un ensemble d'informations qui concernent d'entrée deux types de données référentielles: une grande partie réfère à différentes particularités des produits, leurs gammes, leurs caractéristiques, leurs usages, leurs indications commerciales; l'autre partie porte sur la vie des marques, leur histoire, leur position sur le marché, leurs égéries, leurs codes spécifiques. Au plan de leur manifestation, c'est-à-dire lorsqu'on tente d'en cerner la teneur pour voir quelles déterminations les définissent en tant que spécifiquement dédiés à dire le luxe comme forme d'expression particulière, l'analyse permet d'identifier quatre formes $d^{\prime}$ expression dont le fonctionnement discursif induit des modes d'articulations essentiellement rhétoriques: la légende, le documentaire, l'éloge, la chronique. Toutefois, alors même qu'on s'attendrait à ce que par leur nature ces formes d'expression servent de supports médiatiques pour organiser l'ensemble des discours de ce qui est dit du luxe, le constat est tout autre. Ce sont précisément ces formes qui deviennent l'objet du discours, c'est-à-dire le plan du contenu. Nous n'avons pas affaire à une rationalité sémiologique, où ce qui est donné à voir s'enchaînerait par isomorphisme à ce qui est donné à comprendre. Nous avons affaire en réalité à la mise en place d'ensembles signifiants déjà constitués (une sémiotique) qui de ce fait ne doivent leur portée discursive que du fait de la médiation d'une instance tierce, dans le sens où Éric Landowski ${ }^{4}$ la définit à savoir cette compétence interprétative qui fait de l'objet signifiant non plus une structure déjà là, mais un spectacle auquel on ne peut 4 Landowski Eric, La société réfléchie, Paris, Seuil, 1989. 
accéder que parce qu'on nous en a interprété la signification, autrement ce qui en ressort.

\section{L'instance tierce}

Incidemment, I'on retrouve ici, un des arguments centraux pour souligner l'exclusivité consubstantielle du luxe, face aux autres univers de consommation, comme ce à quoi on ne peut accéder que parce qu'on en connaît les codes:

«ll y a lsidée que le luxe doit être vraiment exclusif, presque intimidant. S`il est trop accessible, cela tue le rêve et cette dimension luxueuse. Les marques ont donc besoin de remettre les consommateurs dans cet esprit-là, de recréer des barrières $»^{5}$,

soulignait notamment dans ce sens Stéphane Truchi, Président du directoire de l'Ifop, interrogé par Relaxnews, une agence française des informations de loisirs, lors du bilan 2014 sur l'état du marché du luxe en France.

Pour le discours, tout dépendra alors de ce que cette instance tierce choisira de traduire, c'est-à-dire de révéler du spectacle. C'est en effet la lecture à effectuer de ces modes d'articulations identifiés. Autrement dit, la légende, le documentaire, l'éloge et la chronique apparaissent au final comme l'effet des jeux rhétoriques de cette instance tierce qui, plutôt que de traduire la substance et l'efficience des marques et des produits auxquels elle a accès, choisit de n'en exprimer que les reliefs ou les reflets. Voilà qui garantit au luxe les conditions de son inaccessibilité, comme réservé exclusivement à ceux qui savent décoder. Tout résulte alors des effets de tri propres à chacune de ces quatre formes de rhétorique. Il s'ensuit une somme de jeux rhétoriques dont le processus sémiosique consiste à ajourner les substances de l'expression, de même que les substances du contenu perceptibles au premier abord.

\section{Jeux de rhétoriques}

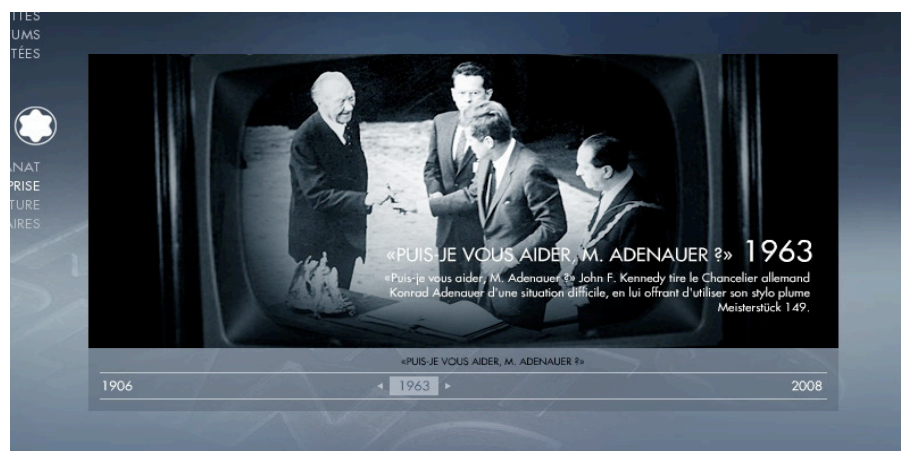

\section{La légende}

5 Voir http://www.ladepeche.fr/article/2013/11/25/1760439-exclusivite-savoir-faire-patrimoine-combinaisongagnante-grands-luxe.html consulté le 15 septembre 2015. 

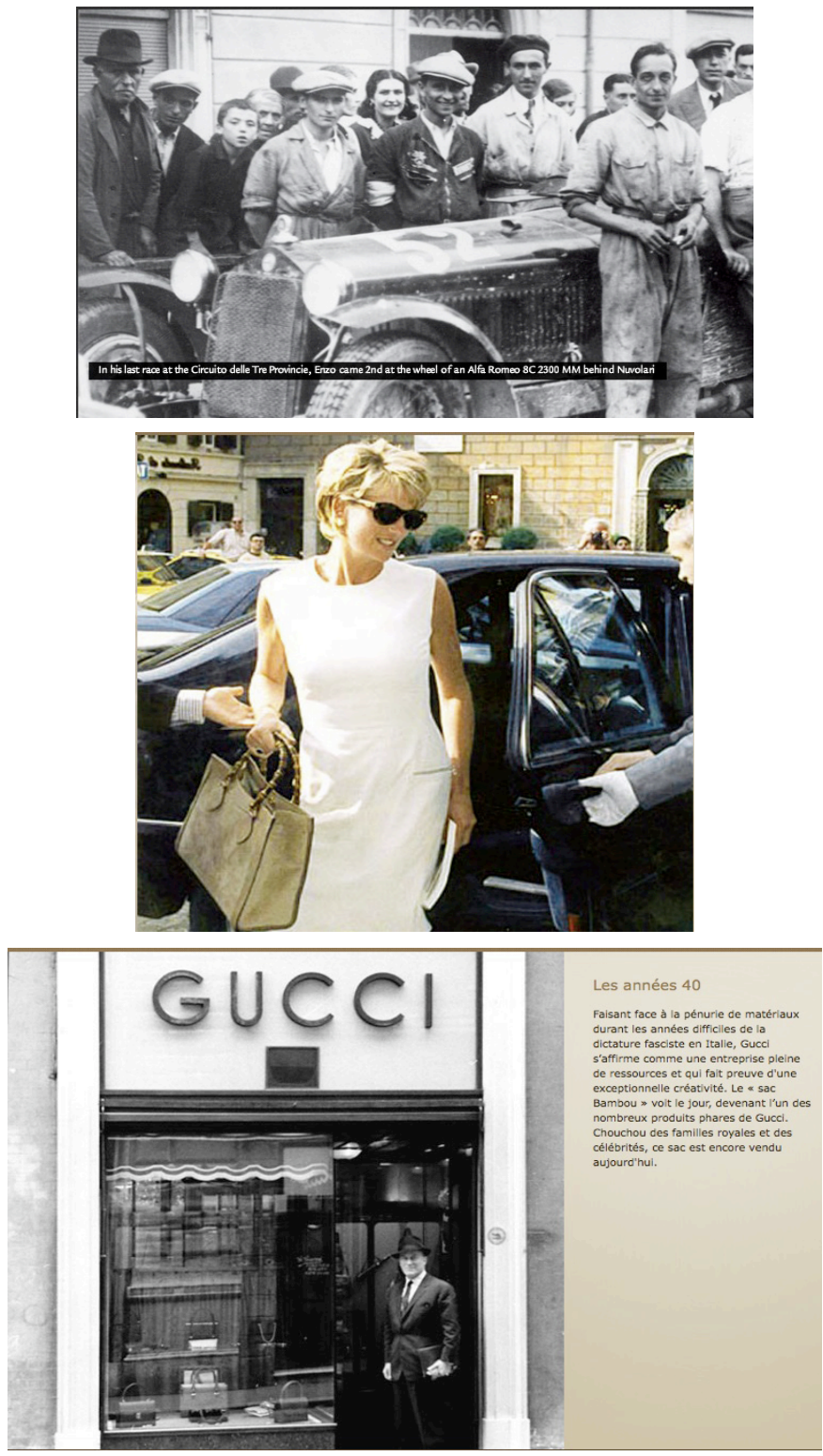

Selon la définition que I'on en dispose, c'est-à-dire en tant que «Récit à caractère merveilleux, où les faits historiques sont transformés par lıimagination populaire ou lıinvention poétique» (Larousse), la légende se distingue en effet par des sauts sémiosiques. Une structure narrative se déploie, et parallèlement, au même moment où ce déploiement 
s'opère, I'histoire racontée perd incidemment en substance, pour ne laisser apparaître que des faits d'articulation («faits historiques transformés», dit le définition), c'est-à-dire les reliefs qui continuent de donner l'illusion (au sens cognitif et cartésien du terme) d'une logique positive de I'histoire. Il en est ainsi des discours tenus sur les sites internet. L'on n'est plus surpris par le pendant exclusivement inductif des péripéties racontées autour des marques et produits. On observe notamment que le narré ne vaut désormais que dans la mesure où il fait de chaque aspect de la marque ou du produit, une évidence qui ne peut que tomber sous le sens de son statut de marque ou de produits de luxe, son histoire, son usage, ses caractéristiques, son inspiration, etc. Ainsi par exemple, tout ce qui précède, tout ce qui a entouré et définit la marque/le produit depuis sa naissance ne trouve son intérêt que comme destiné à en justifier le prestige, la force, le lustre. C'est notamment de ce point de vue qu'il convient de comprendre l'évidente exception de la marque automobile de luxe Ferrari, définie comme liée consubstantiellement au vécu personnel d'Enzo Ferrari. En effet, à lire les informations livrées sur le site internet de la marque Ferrari, le vécu d'Enzo Ferrari, même le plus banal, n’a jamais été qu'exception, à commencer par sa naissance: né le 18 février 1898, contrairement à ce dont on a I'habitude, sa naissance n'a été déclarée que deux jours plus tard à cause d'une forte chute de neige (Exceptionnel!); «A 10 ans, Enzo et son frère Alfredo assistent avec leur père à une course sur le circuit automobile de le Via Emilia à Bologne. Felice Nazzaro gagne la course, mais Vicenzo Lancia réalise le tour le plus rapide du circuit. Le jeune Enzo est totalement subjugué par ce spectacle» ${ }^{6}$. (Exceptionnel!), etc. Et il en est par exemple aussi de John Galliano, pionnier d'une mode révolutionnaire et dont toute la vie n'a jamais été que légendaire. Galliano est né à Gilbraltar, a vécu à Londres, a fait ses études de mode à Saint Martin et il a baptisé le thème de son diplôme de sortie "Les incroyables», inspiré de la Révolution française, annonçant par ce fait même sa propre révolution: le nouveau messie de la mode était né. Toute une histoire!

L'entorse sémiosique se justifie dans la mesure où aucun autre écart n'est possible au regard du contenu inféré à chaque aspect de l'histoire. Le sens est en totalité sous le contrôle d'un contenu (un connotateur) qui est déjà installé comme allant de soi. Dès lors, il ne s'agit plus de construire un signe, d'articuler une signification, il s'agit de témoigner de la manifestation de quelque chose. L'histoire d'Enzo Ferrari de même que celle de Galliano s'apparentent ainsi à ce qu'on appelle aussi une saga, à savoir, selon Régis Boyer, «un récit en prose, [...] rapportant la vie et les faits et gestes d`un personnage, digne de mémoire pour diverses raisons, depuis sa naissance jusqu à sa mort, en n`omettant ni ses ancêtres ni ses descendants s`ils ont quelque importance» ${ }^{7}$

6 http://www.ferrari.com/french/about-us/histoire-de-ferrari/Pages/enzo_ferrari.aspx, consulté le 12 avril 2014.

7 Relevé sur: http://fr.wikipedia.org/wiki/Saga, le 12 avril 2014. 


\section{Le documentaire}
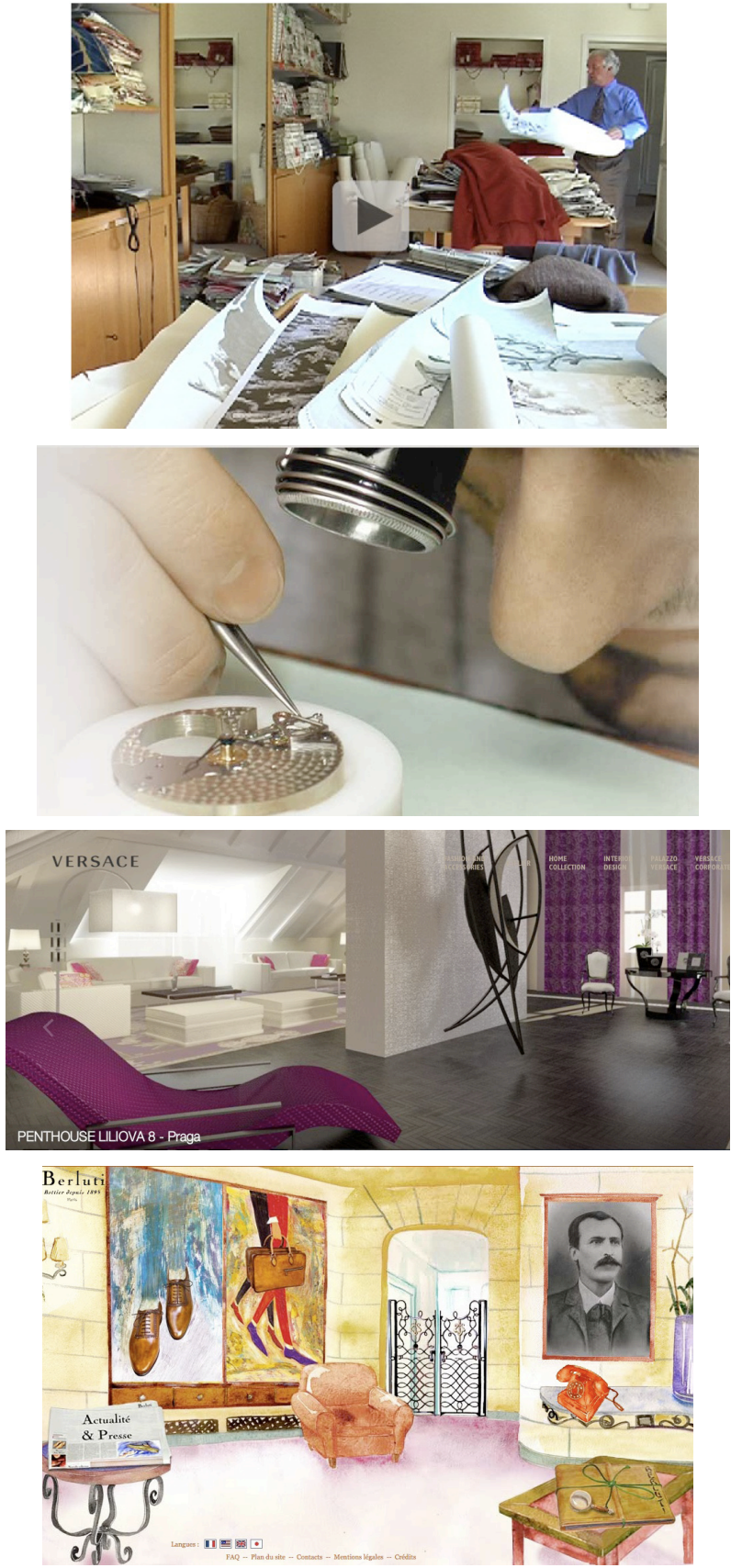
Le même processus est quasi identique pour le documentaire, même s'il ne s'agit pas de narrer. Le documentaire a pour objet, non pas de dire ou de montrer comme cela, en vrac, des éléments, mais de partir de données ayant l'air épars pour construire et affirmer un point de vue, le plus convainquant; I'idée au final étant de persuader que la meilleure interprétation possible est celle qui est présentée. Pour nos énoncés, il s'agit en général de parler des coulisses des marques et des produits pour en confirmer l'excellence, montrer les dessous des produits, ce qui opère de l'autre côté du miroir. Or, ici aussi, du fait même de la définition discursive du documentaire, on observe que tout ne parvient à signifier que parce que contraint: les ateliers, la recherche et développement, l'expertise, le savoir-faire, les secrets de fabrications, etc. ne sont convoqués que dans la mesure où, ensemble, ils contribuent à la perfection de la marque et des produits, sans qu'on n'ait besoin d'en dire davantage. C'est par ce procédé que l'instance tierce parvient à manifester ce qui ressort de l'expression des marques et des produits en tant que lieu d'un spectacle, au sens athénien du terme. C'est un procédé assez récurrent dans l'univers de l'horlogerie par exemple, avec une insistance très souvent appuyée sur l'univers de la recherche et de la création. En général, le discours porte sur la mise en scène du processus de création, on valorise l'expertise, on met à nu la part créative du produit ou de la marque, on recourt à des mises en scène particulières de l'ouvrier ou de l'ingénieur. Souvent aussi, on met en avant un bureau d'études, ou alors on valorise les coulisses des partis pris artistiques, etc. En somme, c'est ici le règne du récit analytique. Ce qui vaut est moins le contenu des signaux retenus pour figurer concrètement la substance du luxe que les effets de discours qui permettent d'inscrire les éléments de rhétorique propre au documentaire: la lenteur, la séquentialisation, l'investigation, mais aussi la rationalisation et l'évaluation, etc. Le contrôle opère par la forme, moins par la substance des contenus déployés.

\section{L'éloge}

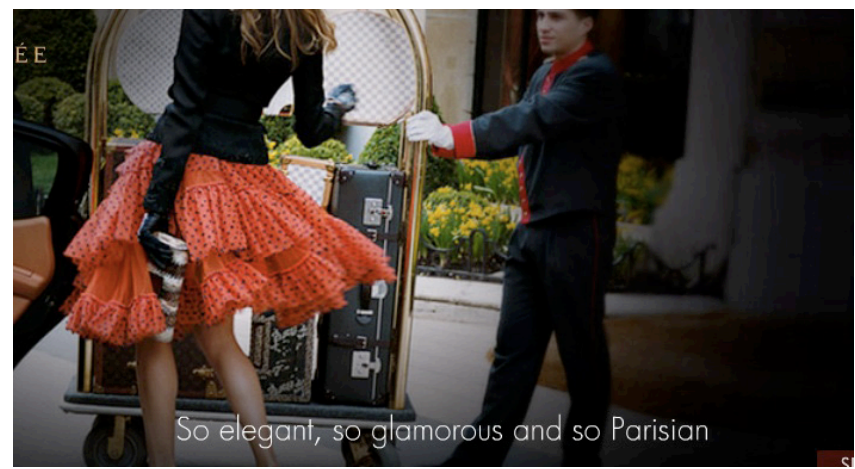



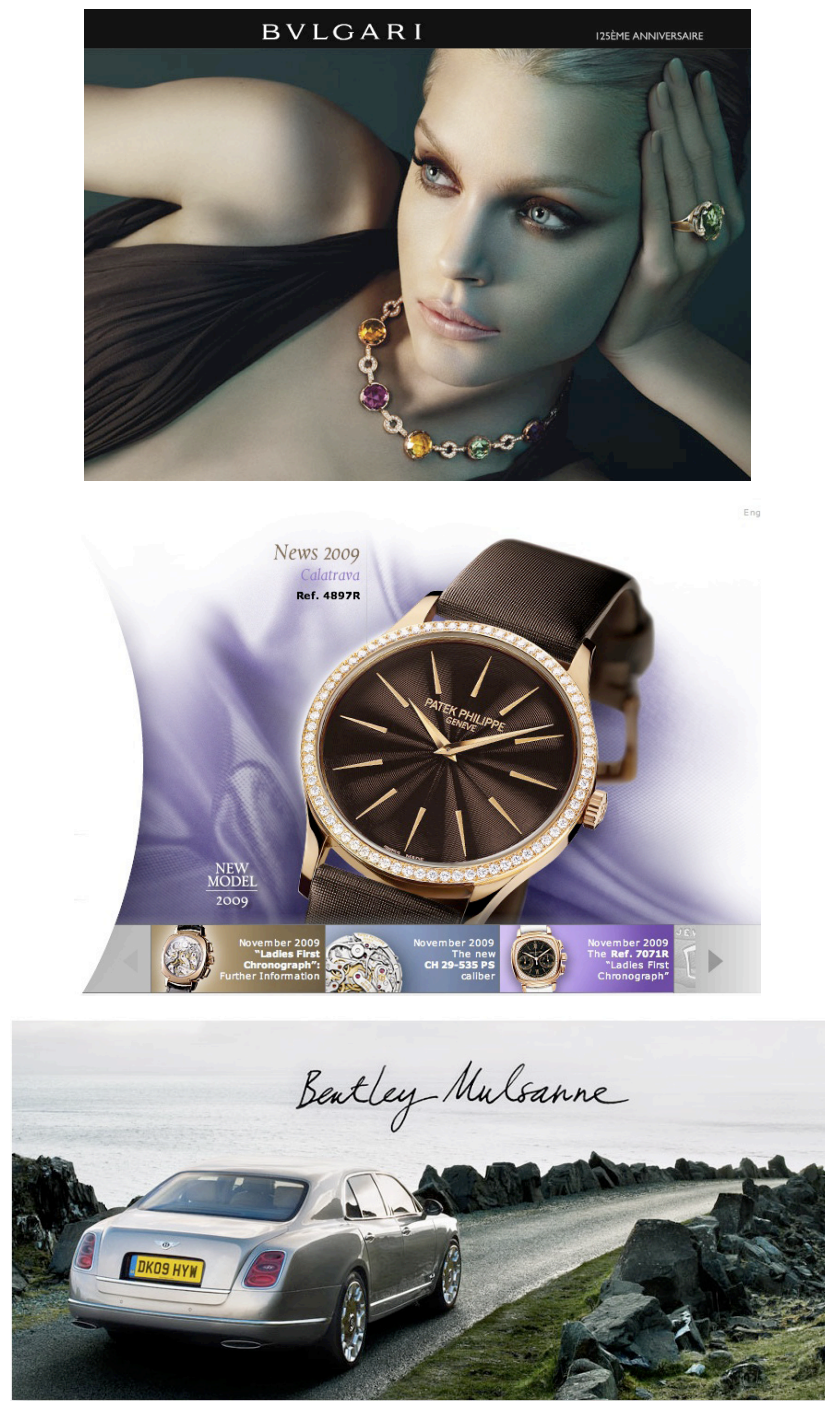

Il s'agit de la forme de contenu qui par elle-même laisse l'impression de donner accès de manière ostentatoire à la substance de la marque ou du produit. Ceux-ci paraissent enfin étalés, accessibles de tous. La mise en scène tire profit des divers procédés de théâtralisation ou de l'exhibition, recours à des halos de lumière, construction d'écrins esthétiques pour magnifier les produits, appel à des discours emphatiques. De manière générale, le style est laudateur, élogieux, avec un usage de superlatifs absolus et une focalisation particulière forte sur l'essence des produits. Or, justement, c'est ici qu'un paradoxe opère, qui conduit à ajourner cette substance. A force de ces faits de théâtralisation et d'exhibition, on observe 
au final que bien que bénéficiant de cette ostentation, au plus précisément à cause de cela, la marque/le produit tendent à ne plus valoir que pour cela, c'est-à-dire perdent en substance. Il s'agit de théâtralisation, d'exhibition, mais dont l'objectif est de mettre sous silence ce qui relèverait des schèmes qui définiraient les marques ou les produits de luxe comme le fait de causalité sémiotique. C'est le théâtre, l'exhibition en tant que tels qui comptent et non plus ce qui est donné à voir instantanément.

Ainsi se justifie l'entorse sémiosique. Attendues pourtant comme une forme contenu pour manifester le sens inféré à la marque et au produit, l'éloge en devient le plan du contenu. On ne voit pas le produit, ce qui le rendrait banal, on voit l'aura, «ce qui fait luxe»: c'est la contrainte, le contrôle.

\section{La chronique}
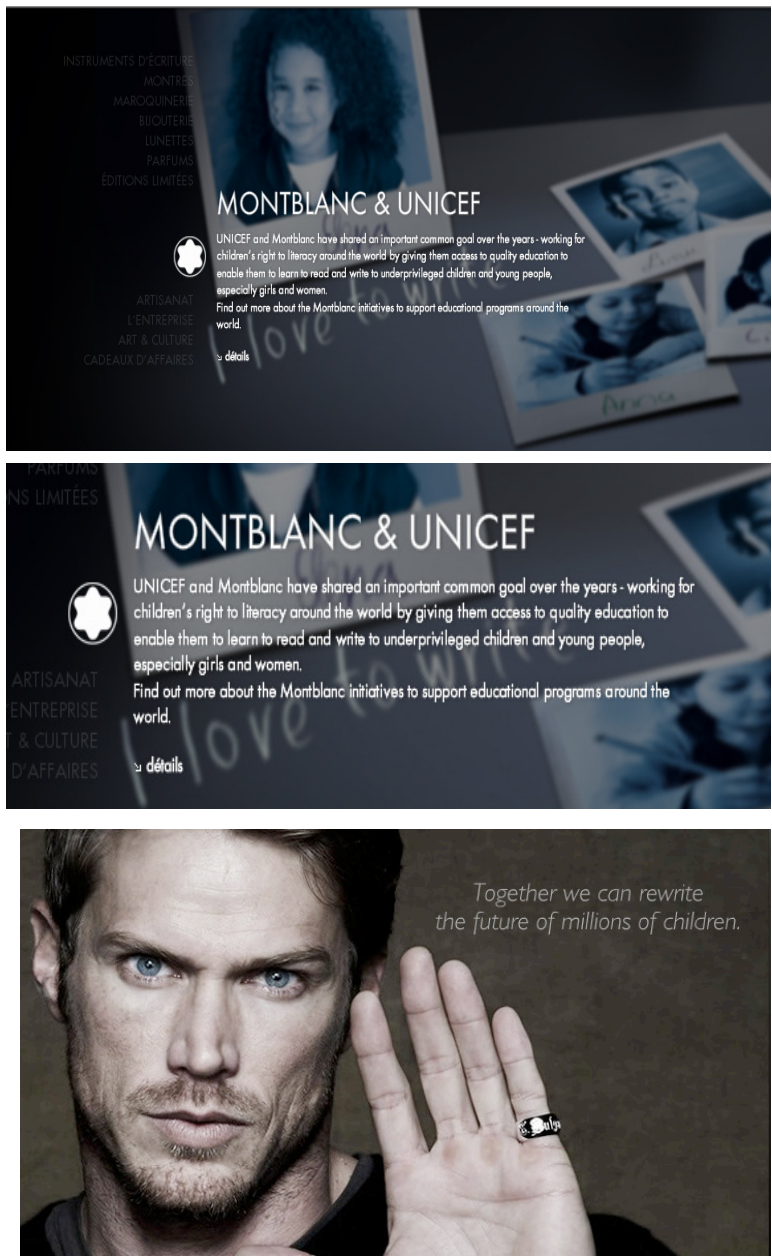


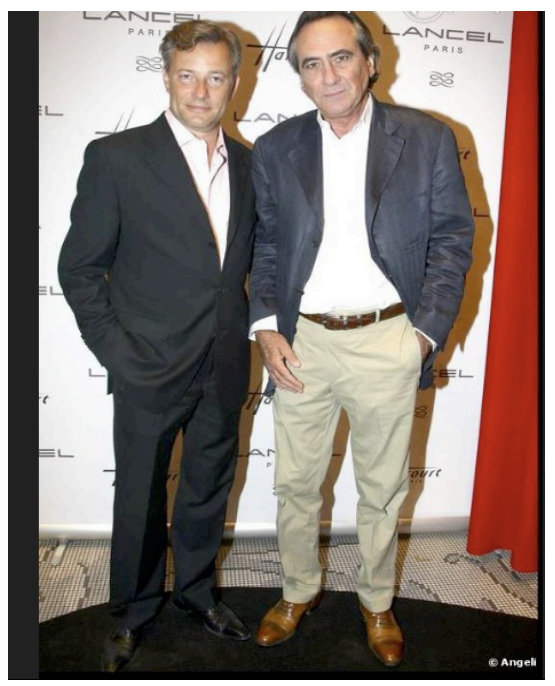

Enfin la chronique. Elle achève de nous installer au cœur de la scénographie la plus évidente de ce qui est en question dans le processus sémiotique à entorse. Il s'agit de spectacle disions-nous. Le modèle d'analyse est alors tout vu. Il renvoie en bien des points à la dramaturgie athénienne dont Eric Landowski donne une schématisation dans son ouvrage La Société réfléchie pour décrire la structure sémiotique de I'opinion. Selon lui, finalement, l'opinion n'est jamais que le fait d'un processus de transformation, pour court-circuiter la parole du public, indistinct, hétérogène, en somme banal. La parole, l'expression du public ne trouve de valeur que reprise et traduite par le chœur qui en observe les péripéties pour les commenter à l'attention des spectateurs. Par la chronique en effet, l'entorse sémiosique opère dans la mesure où en même temps qu'on entreprend de mettre en scène une marque, on la surdétermine par une actualité qui en devient l'objet d'observation.

Ici la mise en scène se sert du temps présent, de l'actualité, des stars du moment pour monter des effets d'affichage et pour montrer l'intérêt existentiel de la marque. Cela passe notamment par une exploitation opportuniste $d^{\prime}$ 'imaginaires reconnaissables ou de faits déjà constitués : des stars de l'actualité, des œuvres caritatives, des célébrités des cautions, des témoignages. Nous citerons l'exemple de diverses actions citoyennes de la marque de stylo Montblanc en lien avec I'Unicef au bénéfice des actions d'éducation à travers le monde; ou encore telle soirée particulière de telle marque. L'idée est de construire des effets d'actualité. Les actes de présence, les coups dans l'air du temps, les faits ponctuels forts est ainsi ce qui devient central ; ce sont eux qui garantissent les contraintes du contrôle: tout élément convoqué, l'est parce qu'il contribue à l'écriture d'un scénario constamment renouvelé et c'est ce scénario particulier qui constitue l'objet du discours, avec la conséquence, comme pour les autres rhétoriques, de l'ajournement de la substance de la marque et du produit. 


\section{Conclusion}

Auparavant exclusif, du fait d'une assimilation un peu trop rapide des classes sociales à tel accès particulier à la richesse, le luxe, en parallèle avec l'éclosion de la consommation de masse, s'est démocratisé, avec une accélération particulièrement intense du fait du développement des moyens de communication virtuels.

Nous avons voulu montrer de quelle manière ces formes de communication opèrent. Nous avons établi la notion d'entorse sémiosique comme étant le principe organisateur de la communication du luxe sur internet, avec la contrainte du contrôle, définis comme ce qui permet d'en préserver l'exclusivité. Alors qu'on aurait dû s'attendre que les rhétoriques pour dire le luxe opèrent comme des formes d'expression, elles en deviennent les formes du contenu : la légende, le documentaire, l'éloge, la chronique. Au final, on retient du luxe non pas ce qui le traduit concrètement, c'est-à-dire la substance des marques ou des produits, mais le spectacle tenu autour de ces traductions. Tel nous a semblé l'enjeu essentiel ; sous le modèle de la théâtralisation. Alors que le média internet offre la latitude la plus large pour faire valoir sous toutes ses formes ce qui d'habitude constitue l'essence du luxe, c'est-à-dire sa substance, tous les jeux rhétoriques opèrent précisément pour ajourner celle-ci. La tactique est toute vue : il s'agit tout en affichant des signes reconnaissables, de les mettre subtilement à distance au seul bénéfice d'une opération préalable de décodage. Le paradoxe du luxe se joue ainsi, comme un ordre discriminatoire.

Didier Tsala Effa é professor de semiótica e comunicação na Universidade de Limoges. Tem uma atuação de mais de quinze anos como consultor de marketing em empresas. É pesquisador do Centre de Recherches Sémiotiques (CeRes), no qual se dedica à semiótica dos objetos do cotidiano com destaque, em particular, ao luxo, aos produtos de consumo massivo e, nos últimos três anos, tem se dedicado ao estudo da robótica humanoide.

didiertsala@yahoo.fr

\section{Références}

Assouly, Olivier. Le luxe. Essai sur la fabrique de l'ostentation. Paris: Édition du regard, 2005.

Courtés, Joseph. Analyse sémiotique du discours. De l'énoncé à l'énonciation. Paris: Hachette, 1991. Fontanille, Jacques. Sémiotique du discours. Limoges: Pulim, 1998.

Pratiques sémiotiques. Paris: Puf, 2008. 
Jolles, André. Les formes simples. Paris: Seuil, 1972.

Landowski, Eric. La société réfléchie. Paris: Seuil, 1989.

Passions sans nom. Paris: Puf, 2004.

Artigo recebido em agosto e aprovado em setembro de 2015. 